\title{
Polymer Thin Film Chemical Sensors
}

\author{
Renat Salikhov ${ }^{1}$ and Aleksey Lachinov ${ }^{2}$ \\ ${ }^{1}$ Bashkir State Pedagogical University, \\ 2Institute of Molecule and Crystal Physics, URC RAS, \\ Russia
}

\section{Introduction}

Polymer and other organic thin films and multilayers are important for a wide range of applications, including electronics, optoelectronics and sensors. Most modern chemical sensors use semiconducting and conducting polymers because they offer cheap technology, mechanical and size advantages (Gerard et al., 2002; Hangarter et al., 2010; McQuade et al., 2000). They can form selective layers in which the interaction between the analyte substance and the polymer leads to change of a such physical parameter as conductivity. Also they can be used in devices that form circuit elements such as transistors (Dimitrakopoulos \& Malenfant, 2002; Qiu et al., 2009). The widespread literature dealing with various applications of these polymers can be divided into two groups: polymers in electronic devices on the one hand (Angelopoulos, 2001) and polymers in chemical sensors based on various transduction mechanisms on the other (Bailey \& Persaud, 2001). The sensor applications take advantage of the physical changes that take place in the polymers when they are exposed to various chemicals. This property has its origin in the molecular and macroscopic structure of polymers. In this paper we shall discuss only sensors based on changing of electronic properties of polymers resulting from their interaction with different chemical agents.

Semiconducting and conducting polymers can be used as the selective layer in sensors or as the transducer itself. For example, change of polymer conductance on exposure to a gas is the sensing mechanism in a chemiresistor. They are relatively easily and inexpensively fabricated. The interaction between organic semiconductor and electrically neutral gas is used as the transduction principle in field-effect transistor (FET) sensors during two last decades (Josowicz \& Janata, 1986).

There are many different formats of chemically sensitive FETs both for gas and liquid applications (Gaponik et al., 1997). In FETs the current magnitude flowing through the polymer is modulated by the interaction with the analyte. The response may depend on both the conductivity and the work function of the polymer. It is difficult to separate influence of the various forms of modulation. For example, in (Polk et al., 2002) localized energy states can affect the value of the work function but do not affect the conductivity of the polymer.

FETs, fabricated with single-walled carbon nanotubes, have been found (Collins et al., 2000) to be sensitive to various gases - for example, oxygen, nitrogen dioxide, ammonia, etc. FETs of this type can operate as gas sensors (Qi et al., 2003) on account of their high sensitivity, their fast response time, and their compatibility with dense-array fabrications. 
Chemically sensitive capacitors (Sundgren et al., 1992) are closely related to FETs. This is not surprising because the FET is a capacitor often fabricated from the same materials and with the same dimensions. Chemically sensitive capacitors, on the other hand, are excited by an applied a.c. voltage, the voltage-dependent capacitance is measured by an impedance bridge and the analytical information is extracted from the shift of the capacitance-voltage curve. Capacitors are obviously easier to fabricate, but in FETs and Schottky diodes, particular attention must be paid to the quality of the contacts between the polymer and the metal.

Most used polymers are p-type semiconductors having rather large values of work function, so the choice of suitable metals to form Schottky contacts is limited. Sensors based on Schottky diodes are relatively simple to fabricate, but the choice of metals is critical and the supporting electronics and data interpretation are more complicated (Potje-Kamloth, 2001)

Semiconducting polymers are useful as building materials in electronic thin film sensors. Because of their porosity they are easily penetrated by gases that can change their electronic properties. Such interactions are advantageous in sensing for gases. Polymer thin films are highly attractive for fabrication of multi-sensing arrays, which makes sensors based on them very suitable for commercial applications. These arrays involve diverse interactive coatings on multiple sensors with the resulting multivariate data analyzed by pattern-recognition techniques. Array detectors are sometimes referred to as electronic noses because the nose uses multiple receptors whose signals are processed by neuronal pattern-recognition processes (Grate, 2000).

In conclusion, polymers are useful materials for gases sensing for several reasons, including their capability to accumulate atoms and molecules on sensor surfaces by reversible sorption and their selectivity due to their chemical structure, which can be easily varied through synthesis. Polymers can yield sensors with rapid, reversible, and reproducible responses. In addition, sets of different polymers can be collected for use in sensor arrays, providing the variable selectivity across the array surface. A sensing polymer must also have a number of desirable physical properties. For example, solubility in organic solvents may be necessary, or a certain viscosity for using coating processes.

\section{Polyarylenephthalides (diphenylphthalide-based polymers)}

Over the last decades, a new scientific area has been developed, the electronics of thin films of electrically active polymers. The possibility of creating organic films, including polymer ones, with fantastic physical properties exists. With decreasing the material thickness below a certain value, various factors, previously insignificant, start affecting the appearance of new properties (including electronic ones), the surface effect increases significantly. This manifests itself especially strongly in the case of metal - insulator contacts. In what follows, it was not distinguished the concepts of an insulator and a polymer, because it will be considered polymers having a wide energy gap $E_{g}\left(E_{g}>2 \mathrm{eV}\right)$. The main paradox of the phenomena described in this review consists in the fact that the efficient charge transfer (electric conductivity) occurs in materials all of whose properties indicate that they are insulators.

In the early 1980s, a new polymer-poly(diphenylenephthalide) (Salazkin et al., 2008)-was synthesized at the Institute of Organic Chemistry (Ufa Research Center, Russian Academy of Sciences), which opened the way to synthesizing a number of new compounds. Its structural formula and optimized three-dimensional geometry are shown in Fig. 1, 9. 
As was established later using the method of quantum-chemical calculations (Johansson et al., 1994), a remarkable feature of these polymers is that with a change in the length of one of the bonds in such a molecule to a certain magnitude, a second energetically stable state can arise that is characterized by the existence of electron levels in the region of previously forbidden energies. To perform quantum-chemical calculations of the polymer structure the semiempirical Austin Model 1 (AM1) method (Dewar et al., 1985) was used to optimize the chemical structure of organic molecules and the pseudopotential method of the valence effective Hamiltonian (VEH) (Bredas et al., 1981) was used with the data obtained in terms of the AM1 method as the initial parameters. The authors of (Johansson et al., 1994) studied properties of an isolated chain, although it is obvious that taking the intermolecular interactions into account may be important for the understanding of the relevant phenomena. In all cases, the geometry was optimized using monomer or dimer units, whereas the electron structure was determined for periodic boundary conditions with a monomer unit. Calculations in terms of the VEH method yield the electron energy band structure.

Figure 1 shows the optimized geometry for three different cases. In a neutral system (Fig. 1 a), the central carbon atom in the backbone chain is quaternary ( $\mathrm{sp}^{3}$ hybridization), and the $\mathrm{C}_{6} \mathrm{H}_{4}$ group (which must enter the main chain of the polymer) is in the benzenoid state. Benzenoids are monocyclic aromatic compounds with delocalized $p$ electrons, because $p$ bonds in the cyclic group are conjugated. Moreover, the distance from both phenyl groups (in the main chain) to the central quaternary carbon atom turned out to be $1.50 \AA$. The distance between the central atom and the nearest oxygen atom in the side group (C - O spacing) is equal to $1.47 \AA$. The torsion angle between the planes of phenyl groups inside the monomer is about $65^{\circ}$, which indicates a weak conjugation degree along the polymer chain.

The calculation started from the initial geometry in which the central carbon atom was in the $\mathrm{sp}^{2}$ hybrid state, the bonds surrounding the carbon atom lay in the same plane, and the oxygen atom was not bound to the central carbon atom. The procedure of optimizing the molecule geometry was stopped at a local minimum with a slightly increased formation heat compared with that of the neutral ground state. The phenyl groups retained the benzenoid type. The structure around the central carbon atom was contracted, which led to a decrease in the length of the bonds. The distance from the carbon atoms of the phenyl groups to the central carbon atom was now about $1.44 \AA$, whereas in the geometry of the neutral ground state, it was equal to $1.50 \AA$. The C - O spacing in this case was $3.10 \AA$. A separate preliminary calculation of the reaction coordinates for isolated polymer chains showed that the magnitude of the activation barrier is not more than the actual difference in the heats of formation of these two optimum configurations. The local energy minimum is very shallow; therefore, the shape with an open cycle in the neutral molecule is very unstable. However, intermolecular interactions, including those caused by the dipole moment, reduce the attraction between the positive carbon atom and negative carboxyl group and lead to a stabilization of the chemical structure. This apparently indicates that the shape with an open bond can be formed as a result of the action of external forces in the solid state, when the surroundings of the polymer chains are capable of stabilizing the higher energy state.

The addition of an electron and of a compensating ion to any of the three geometric configurations under consideration shows that the structure with an open cycle becomes stable. The energy of stabilization of the monomer is about $113 \mathrm{kcal} \mathrm{mol}^{-1}$. The bond lengths indicate that the phenyl group continues to be of the benzenoid type as before. The largest change is observed in the distances from the phenyl groups to the central carbon atom, which decrease from 1.50 to $1.43 \AA$. The bond lengths along the polymer chain become almost equal 
to each other. It can be seen from Fig. $2 \mathrm{c}$ that the $\mathrm{C}-\mathrm{O}$ bond is broken (the $\mathrm{C}-\mathrm{O}$ spacing is equal to $3.65 \AA$ ). This suggests that the central carbon atom has the $\mathrm{sp}^{2}$ rather than $\mathrm{sp}^{3}$ hybridization, as in the neutral state. We note for comparison that the opening of the $\mathrm{C}-\mathrm{O}$ bond is a well-known phenomenon in the case of phenolphthalein, which has a chemical structure resembling the structure of the monomer of the polymer under consideration.

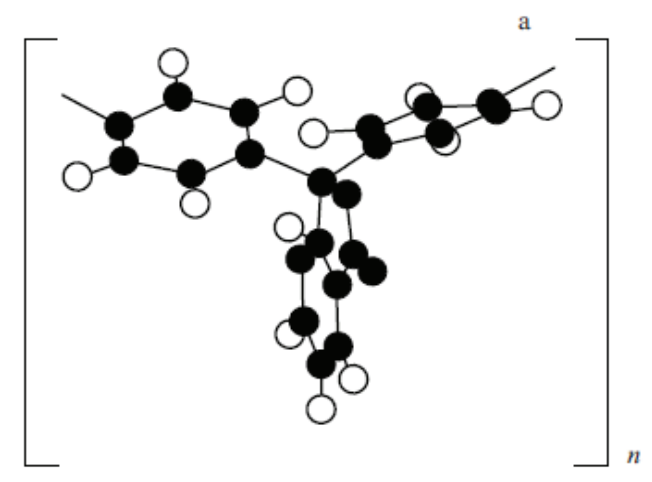

b
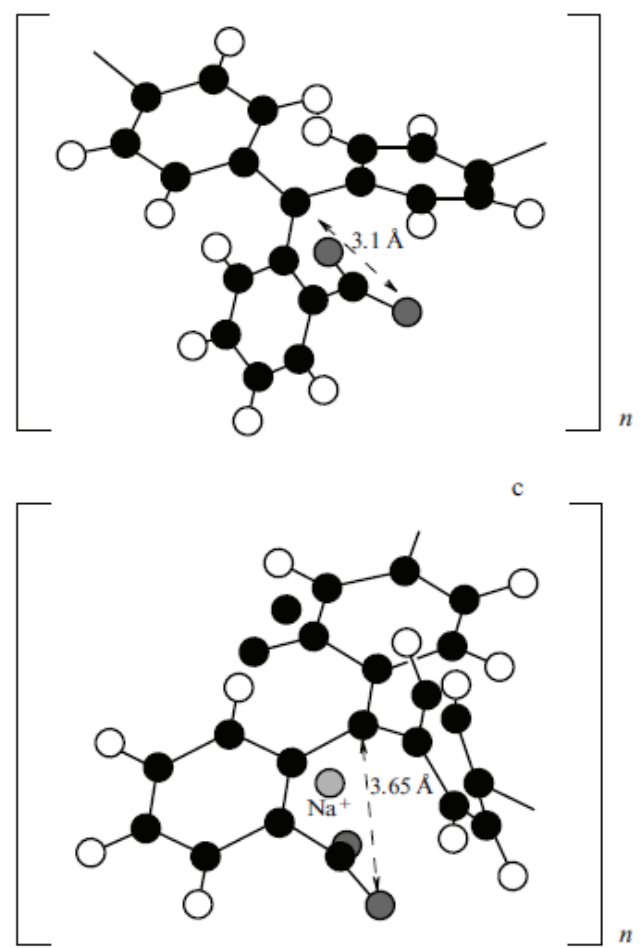

Fig. 1. Optimized geometry for three different cases: (a) a neutral molecule in the ground state; (b) the molecule in the state with a greater total energy, which corresponds to an open lactone cycle and (c) alloying by adding one electron and a counter-ion $\mathrm{Na}^{+}$to the molecule 
The investigation of geometric optimization with a monomer used as the unit cell shows that two different geometric structures with a relatively small difference in the total energy of the ground states can be realized in polyarylenephthalides. Thus, numerical investigations have shown that the individual molecules of poly(diphenylenephthalide) (PDP) have two energetically stable geometric configurations. The first corresponds to the neutral ground state, which is characterized by the widths of the energy gap between the top occupied and bottom vacant orbitals equal to $4.2 \mathrm{eV}$. The second configuration is determined by a modified set of lengths of intramolecular bonds and a nonzero spectrum of electron states in the region of forbidden energies. However, the stability of this state is insufficient to ensure its actual existence. But its stability increases (the local minimum becomes relatively deep) if the molecule captures an electron and becomes a negative-charge ion. In this case, the calculations predict the appearance of a new deep electron level in the energy gap.

However, the results of calculations based on the experimental data on measurements of the energy characteristics of the polymer by X-ray photoelectron spectroscopy (XPS) (Zykov et al., 1992) were insufficient to correctly understand the properties of this material.

In the language of the energy band model, this process must lead to the appearance of deep electron levels located, according to the calculations in (Johansson et al., 1994), almost at the Fermi level. It follows from these calculations and experiments that by changing the rate of injection of charge carriers into the polymer film, one can substantially control the electric conductivity of wide-band nonconjugated polymers. The available experimental work (Lachinov et al., 1990; Kornilov \& Lachinov, 1997) generally confirmed this conclusion.

Optical properties of poly(diphenylenephthalide) films near the electronic switching threshold have been studied by modulation spectroscopy (Lachinov et al., 2010). The results obtained have been interpreted using quantum chemical calculations of the electron energy characteristics of the polymer molecule. It has been established that the injection of charge carriers into the polymer film and interaction of this excess charge with the macromolecule can lead to the formation of deep trap states near the center of the band gap.

The electroabsorption modulation spectra were obtained for the PDP films at different amplitudes of the modulating voltage. The spectra consist of two bands. At a voltage of 0.5 $\mathrm{V}$ across the sample, the maximum of the first band is located in the range $490-515 \mathrm{~nm}$ and the maximum of the second band is located in the range 580-610 $\mathrm{nm}$. An increase in the voltage changes the shape of the bands and positions of their maxima. At a voltage of $0.8 \mathrm{~V}$, the intensity of the first band increases and the position of the maximum is shifted toward the long-wave spectral range. In this case, the intensity of the second band slightly decreases and the position of its maximum is shifted toward the long-wave range.

It was assumed that the modulation of absorption is associated with the presence of electronic states in the mobility gap of the polymer (Fig. 2). The same states can participate in the transfer of the charge injected into the polymer from electrodes. According to (Lachinov et al., 2006), the conductivity in a thin polymer film is caused by the appearance of a narrow charge transfer band located near the center of the band gap of the polymer. In this case, the modulation of optical absorption results from the change in the population of deep electronic states by injected charge carriers. The occupation of the states leads to a decrease in the absorption, and their depletion results in an increase in the absorption. It is worth noting that the position of the specific features in the first region of the modulation spectrum coincides well with the position of the emission bands in the photoluminescence and electroluminescence spectra of PDP. 


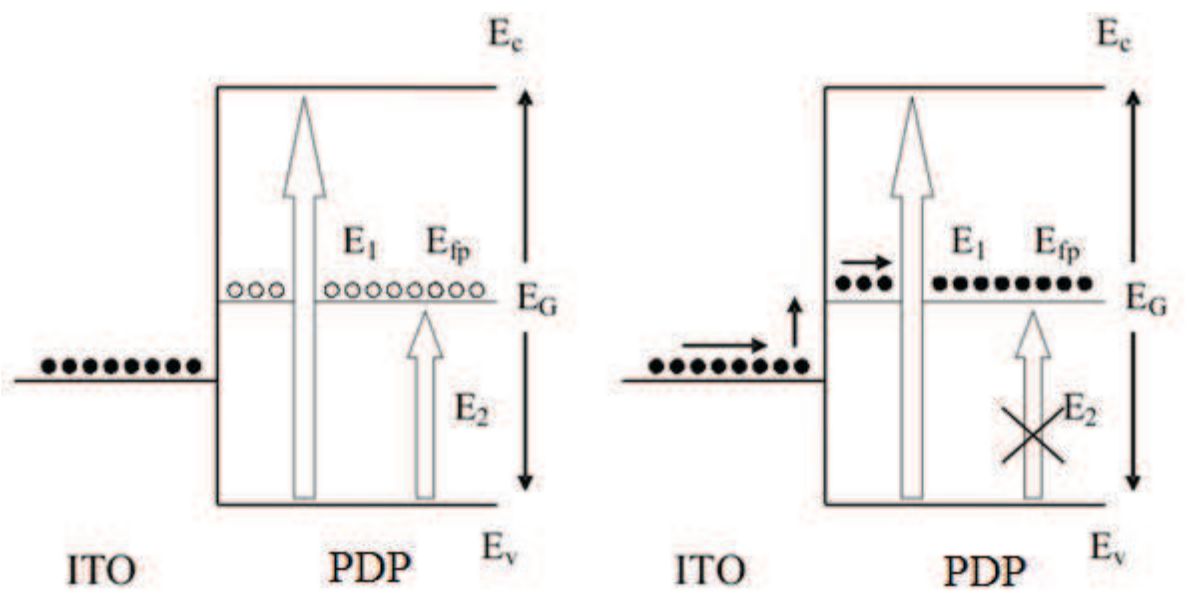

Fig. 2. Band diagrams of the ITO/PDP interface for (a) vacant and (b) occupied electronic states in the polymer film: $E_{f p}$ is the Fermi level of the polymer, $E_{C}$ is the conduction band, $E_{V}$ is the valence band, $E_{1}$ is the transition from the valence band to the conduction band, and $E_{2}$ is the transition from the valence band to the narrow charge transfer band.

Thus, it was established using modulation spectroscopy that the charge injection into the polymer film near the electronic switching threshold leads to the formation of the additional structure in the absorption spectrum in the transparency range of the polymer. The spectral position of the new structure correlates well with the position of maxima in the photoluminescence and electroluminescence emission spectra. The analysis of experimental data with due regard for our quantum chemical modeling of the interaction between the excess electron and the polymer molecule within one monomer unit demonstrated that this interaction can result in the appearance of new deep electronic states. The energy position of these states agrees satisfactorily (with allowance made for a relatively large error in the calculation) with the results of measurements of the electroabsorption.

Complex investigations permitted revealing many new physical phenomena in organic polymeric objects with a nontraditional conductivity. To emphasize the unusual character of such materials, it is convenient to call them electroactive, in contrast to well-known electroconducting materials, which are mainly conjugated polymers. This means that most electroconducting polymers have double bonds in the molecule structure, whose reactivity (and, consequently, energy) depends on the number of such bonds (i.e., these bonds affect one another), whereas in polymers based on diphenylphthalide, the active double bonds of the different parts of the molecule are independent of each other.

The transition from a dielectric state to a high conductive state (HCS), induced by abnormal low external influences is observed in thin electroactive polymer (polyheteroarylines) films. The feature of this phenomenon is that the temperature dependence of the HCS conductivity is of the metal type and the conductivity can be extremely high $>10^{5}(\Omega \cdot \mathrm{sm})^{-1}$. Also polymer films in the HCS possess a high anisotropy of conductivity. In the HCS the polymer film reveals a domain pattern: insulating matrix surrounds conductive domains. The domain diameter ranges from 25 to $1000 \mathrm{~nm}$ (depending on the polymer film thickness). The main effects of electron switching were considered in sufficient detail in (Lachinov et al., 2006). 
The unusual properties of the thin polyheteroarylines films open broad prospects for their application in variety of industrial and technological branches. Mechanical sensors can be based on the pressure dependence of the polymer film conductivity. In switches, keyboards and digitizers the high sensitivity of the polymer film to uniaxial pressure can be used. The effect of switching from the metallic to the insulating state when the current exceeds a critical value can be applied for protecting electric circuits from current overload. Since a working element of such a device is a single conducting domain of $\approx 1 \mu \mathrm{m}$ diameter, it can be formed as part of an integrated circuit. The polymer compound can be utilized as glue with anisotropic conductivity for surface assembly instead of soldering in the electronics industry. The effect of bistable switching in an electric field can be used for producing devices similar to ovonic memory switches. Temperature sensors can be based on the effect of thermostimulating switching. Pristine polymer film in the HCS can be used as a substitute for precious metals to protect contacts in various switches.

\section{Charge transport in poly(diphenylenephthalide) thin films}

Currently there is great interest in the use of polymers for the fabrication of various electronic devices (Halik et al., 2004; Salleo et al., 2004) due to the significant processing, mechanical, and size advantages. As compared with inorganic materials, polymers exhibit a greater variety of properties related to charge transport mechanisms offering more ways to improving performance. Most devices use silicon/polymer/metal structure (Musa \& Eccleston, 1999), which also suits the goals of our study. Keeping in mind possible applications we chose $\mathrm{Si} / \mathrm{PDP} / \mathrm{Cu}$ "sandwich" type structure to carry out current-voltage (I$V)$ characteristic measurements in a range of temperatures to identify the predominant current transfer mechanisms and further elucidate the electronic structure of the polymer under investigation (Salikhov et al., 2007a, 2007b).

Organic-inorganic multilayer structures of the silicon/polymer/metal type have been fabricated. The current-voltage characteristics of these structures versus temperature are measured. The obtained results are treated within different models of charge transport such as Richardson-Schottky thermionic emission, Fowler-Nordheim tunneling, and Pool-Frenkel and hopping mechanisms. The conclusions about the mechanisms of charge transport at different electric fields and temperatures have been made. The Richardson-Schottky model is in good quantitative agreement with the experimental data in the case of the metalpolymer interface with the energy barriers of $0.26-0.10 \mathrm{eV}$. The charge transport in the polymer is explained by the hopping mechanism near the Fermi level with the activation energies being of $0.01-0.04 \mathrm{eV}$.

$\mathrm{I}-V$ characteristics of the three-layer $\mathrm{Cu} / \mathrm{PDP} / p-\mathrm{Si}$ structure have been measured over a range of temperatures. The data obtained were analyzed by fitting theoretically predicted dependencies of conductance on the electric field to the experimental ones. Relevance of the corresponding model was then assessed by comparison of the fitting parameters with the measured activation energies and the other polymer properties. It was concluded that the dominant charge injection mechanism, at least at higher temperatures, is the thermionic emission. Also, it was shown that the tunneling through the contact-polymer interface gives no significant contribution to the charge injection into the film. It was also concluded that the charge transport through the film thickness may be satisfactorily explained by the hopping of carriers between localized states in a narrow energy band near the Fermi level. 
In a series of works, Bassler and his colleagues (Bassler, 1993; Richert et al., 1989) developed a detailed model of charge carrier transfer. The organic matrix with embedded transfer centers is simulated by a cubic lattice with a lattice constant $a$ in which each site is occupied by a transfer center. The position of the energy level $E$ involved in the charge transfer for each center is an independent random quantity due to the stochastic and uncorrelated influence of the environment. The corresponding density of states is described by a Gaussian distribution,

$$
\rho(E)=\left(2 \pi \sigma^{2}\right)^{-1 / 2} \exp \left(-E^{2} / 2 \sigma^{2}\right) .
$$

This choice of the density of states $\rho(E)$ is additionally confirmed by the Gaussian shape of the absorption and fluorescence bands experimentally observed for many polymers. The specific calculations within the described model were performed using only Monte Carlo numerical simulation. It was revealed that the field dependence of the mobility is an $S$ shaped curve. The central portion of this curve is approximately described by a linear dependence of the quantity $\ln \mu$ on $F^{1 / 2}$. The results of these calculations made it possible to propose the model of hopping transport over centers with a Gaussian distribution of energy levels. Analysis of the data obtained in our work can affirm the aforementioned hopping model of charge transfer in the temperature range preceding the transition to the high conductivity state (Salikhov et al., 2007c, 2007d).

Considerable attention has been paid recently to analysis of the properties of the contact between two organic materials. This is primarily due to the need for matching the energy parameters of individual contacting layers in multilayer systems of the type of organic electroluminescent diodes. Transport properties of a multilayer system containing such an interface are considered as a rule in the direction perpendicular to the interfacial plane, which can be explained by the type of charge transport in an external electric field. However, it should be noted, that there have been problems associated with the inability to obtain sufficient mobility of charge carriers necessary for practical applications.

\section{Conducting polymer/polymer interface}

The properties of a near-surface region in films are usually different from those in the bulk. This difference is related to several factors, including the excess free Gibbs energy of the surface and a decrease in the entropy. The mutual influence of contacting layers takes place at their interface (boundary) and involves both the structure of a transition layer and its electron properties. Changes in the electron properties can be traced by measuring the transport characteristics of the resulting two-dimensional (2D) structures. However, the study of the electron transport properties of boundary layers is frequently complicated by a relatively high intrinsic conductivity of the initial polymers, which masks the conductivity of the boundary layer. Therefore, for the experimental investigation of such systems, it is expedient to select polymers with minimum intrinsic conductivity (e.g., dielectrics), so as to minimize the current component passing through the volume of polymers and study a change in the conductivity along the contacting layers.

Recently it was reported about the interface between two insulators $\mathrm{LaAlO}_{3}$ and $\mathrm{SrTiO}_{3}$ with anomalous electronic properties, in particular, the charge carrier mobility $\mu=10^{4} \mathrm{~cm}^{2} \mathrm{~V}-1 \mathrm{~s}^{-1}$, was investigated (Ohtomo \& Hwang, 2004). Later on (Reyren et al., 2007), superconductivity and giant magnetoresistance were revealed in such structures. These phenomena were 
explained in the framework of the hypothesis that a two-dimensional electron gas is formed in the transient layer between two insulators. The mechanism of the formation of this electron gas is not clearly understood, but active studies made it possible to reveal many interesting properties of this interface including superconductivity at $200 \mathrm{nK}$, magnetoresistance, ferromagnetic ordering of the electrons, field control, and many other properties inherent in a two-dimensional electron gas. In 2008, similar results were obtained in (Alves et al., 2008), where the transient layer was formed at the interface between two organic insulators -tetrathiafulvalene (TTF) and 7,7,8,8, tetracyanoquinodimethane (TCNQ). The mobility in these structures was about a few $\mathrm{cm}^{2} \mathrm{~V}^{-1} \mathrm{~s}^{-1}$, and the metal conductivity was revealed. At the same time anomalously high conductivity at the interface between two identical polymer layers of poly(difenylenephthalide) has been demonstrated (Salikhov et al. 2008).

This paper presents the results of further investigation of the charge transport along the boundary of two organic layers of PDP (Gadiev et al., 2010; Salikhov et al., 2009). It was expected that the bending of energy levels in this region must lead to increased local charge concentration and, hence, modify the transport properties of the $2 \mathrm{D}$ structure under consideration. In particular, the electric conductivity of the interface layer must be several orders of magnitude larger than the bulk one. In this connection, it seems important to study the electronic properties of the interface between two dielectric polymer films. The objective of this work was to form the polymer-polymer interface and to investigate its electrophysical properties including the sensory ones.

We used poly(diphenilenephthalide)s of the polyheteroarylene polymer class as the objects of investigation. These polymers are highly soluble in organic solvents and possess high film-forming properties. In our experiments, polymer films were obtained by spin coating of the polymer solution in cyclohexanon of various concentrations. The film thickness varied from $30 \mathrm{~nm}$ to $1 \mu \mathrm{m}$. Metallic copper electrodes were prepared by vacuum thermal-diffusion sputtering. The current-voltage $(I-V)$ characteristics of the samples were measured using the standard technique. Temperature measurements were taken in a range of 90-300 K using a cryostat based on a Dewar vessel. The system under investigation was placed in the Dewar vessel, and the temperature was varied by changing the position of the sample relative to the nitrogen surface. Temperature was monitored with the help of a thermocouple.

The choice of the polymer material was dictated by the following considerations. Analysis of electron transport properties of the boundary layers proper is complicated, since conducting polymer materials possess a high intrinsic conductivity, which may shunt the conduction of the boundary layer. In this connection, measuring conditions must be ensured such that the conductivity along the interface being studied is higher than the bulk conductivity of the material. Consequently, for our experiments we chose polymers with a low intrinsic conductivity. The optical band gap of the polymers used is $3.9-4.2 \mathrm{eV}$ and the electron work function is approximately $4 \mathrm{eV}$. Obviously, such polymers are poor conductors in the initial state. For this reason, any insignificant improvement of their transport properties can easily be monitored.

The experimental samples were the multilayer structures presented schematically in Fig. 3a. The sample was prepared by consecutive operations as follows. The polymer film was 
deposited on the surface of the purified polished glass substrate by spin coating from the PDP solution. The solvent residuals were removed from the film by successive drying first in air at room temperature for $30 \mathrm{~min}$ and then at $150^{\circ} \mathrm{C}$ for $40 \mathrm{~min}$. Then, the metal electrodes were deposited on the polymer film surface by vacuum thermal diffusion sputtering through the shadow mask. The final stage was the deposition of the second polymer layer adhering the entire sequence of technological operations described above. Each layer was about $300 \mathrm{~nm}$ thick. The thickness of polymer films and the state of the surface were monitored by a SMM-2000T atomic force microscope. The electrodes were made of copper. The distance between the electrodes was varied from 30 to $60 \mu \mathrm{m}$.
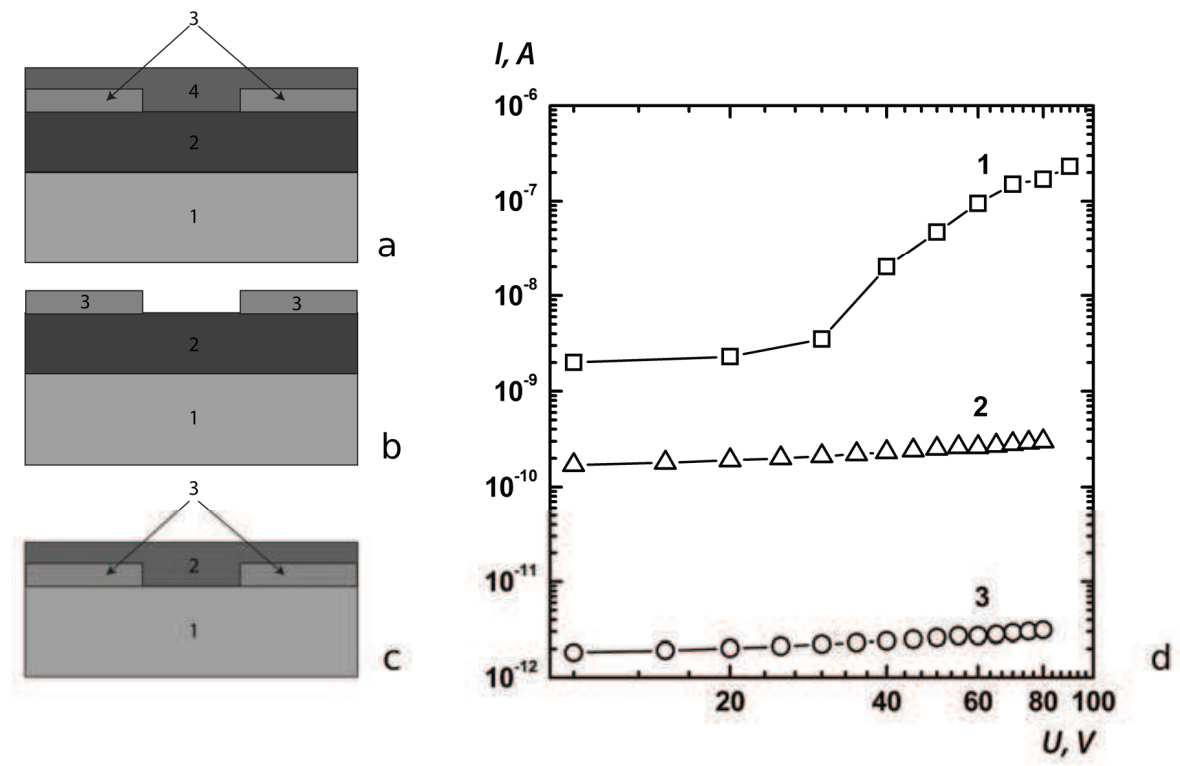

Fig. 3. Structures of the experimental samples with (a) two polymer layers, (b) one polymer layer and the electrodes on the polymer surface, (c) one polymer layer and the electrodes under the polymer film, where 1- substrate, 2- polymer layer, 3- metal electrodes, 4- polymer layer, and (d) current-voltage characteristics for samples: (1) - a, (2) - b, and (3) - c

In the preparation of the sample, the resistivity was controlled by the two-probe method. As soon as the electrodes were deposited on the first film surface (see Fig. $3 b$ ), the resistivity was $\rho \sim 10^{7} \Omega \cdot \mathrm{cm}$ (Fig. 3d, curve 2) and decreased down to $\rho \sim 10^{3} \Omega \cdot \mathrm{cm}$ when the second layer was deposited (see Fig. 3d, curve 1). To refine the possible contribution of the charge carrier transport along the polymer-substrate interface, the samples with the electrode on the glass substrate surface were additionally prepared (see Fig. 3c). In this case, the resistivity was $\rho \sim 10^{8} \Omega \cdot \mathrm{cm}$ (see Fig. 3d, curve 3). An analysis of the currentvoltage characteristic obtained in the measurement of the charge carrier transport along the polymer-polymer interface (see Fig. 3d, curve 1) demonstrated that it is well approximated by the function of the form $I=k U^{n}$. Such dependences can be interpreted in the injection model. This conclusion does not contradict the results of the earlier studies of the electron transport properties of the metal-poly(diphenylphthalide)-metal system [10]. 
Two regions with different exponents $n$ are clearly seen on the current-voltage characteristic: $n=1$ at low voltages, and the current-voltage characteristic at higher voltages is superlinear with $n \sim 2$. The voltage at which the transition from the linear to the superlinear region occurs corresponds to the situation when the concentration of the thermally generated equilibrium free charges becomes comparable to that of the injected charges (Bunakov et al., 2003). This balance of concentrations allows us to estimate the electron mobility $\mu$,

$$
\mu=J L^{3} / \theta \varepsilon \varepsilon_{0} U^{2} .
$$

The current density $J$ was estimated in terms of the current and the surface area through which the current was flowing, and which, in turn, was calculated in terms of the electrode width and the transport layer thickness; $\theta$ is defined as the ratio of the maximum and minimum currents of the limiting trap filling region. According to the AFM recording (see Fig. 4), the transport layer thickness was set equal to $10 \mathrm{~nm}$ and $U$ is the voltage at the point of the transition from the linear to the square law. The charge carrier mobility calculated by formula (2) was $3.76 \cdot 10^{-2} \mathrm{~cm}^{2} \mathrm{~V}^{-1} \mathrm{~s}^{-1}$. It is very high for such materials and is almost five orders of magnitude higher than the bulk mobility $\mu \sim 10^{-6} \mathrm{~cm}^{2} \mathrm{~V}^{-1} \mathrm{~s}^{-1}$ (Lachinov et al., 2004) measured by the time-of-light method. The value obtained is close to the maximum charge carrier mobilities in organic materials, in particular, in organometallic compounds such as phthalocyanines $\left(\mu \sim 0.4 \div 1 \mathrm{~cm}^{2} \mathrm{~V}^{-1} \mathrm{~s}^{-1}\right)$.

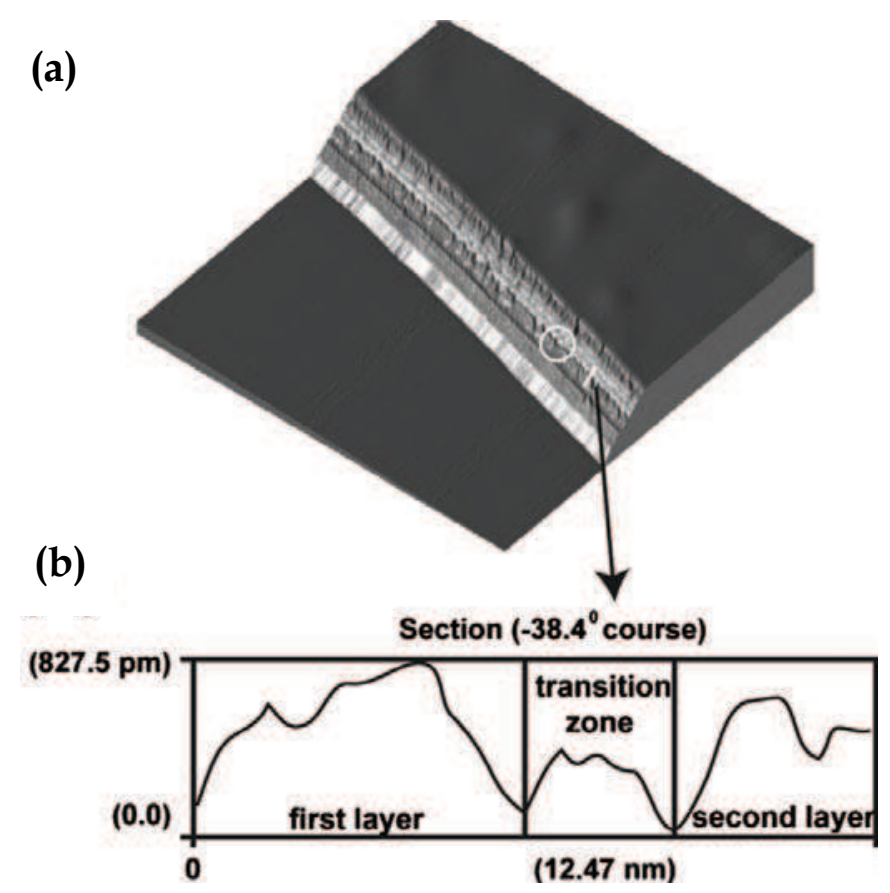

Fig. 4. The interfaces between two polymer layers (atomic-force image). a) 3D image of the two-layer polymer film, (polymer/polymer interface marked by a white circle). b) 
Transition zone profile between two polymer films (the same area at the 3D image marked by a white line).

It is well known that the bulk conductivity of the polymers studied here is of the hole type. At the same time, the formation of the highly conducting state is associated with electron conduction. The question arises about the type of charge carriers ensuring an elevated conduction of the boundary transport layer. The answer to this question could be obtained from analysis of transport characteristics of transistor structures. Thus, we consider the results of measurements of the $I-V$ curves for samples prepared on silicon plates with an arrangement of electrodes similar to the configuration of a field transistor (see Fig. 5a). Samples with polymer films on silicon plates with an oxide layer were prepared using an analogous technique. The substrates for the transistor structures were $n++-S i$ plates with an ohmic electrode on one side of the plate and a $\mathrm{SiO}_{2}$ layer (100 nm in thickness) formed by thermal oxidation on its other side.

We measured the dependence of the current between the drain and the source (lateral electrodes) on the potential supplied to the gate (lower ohmic electrode on the silicon plate) (Fig. 5b). The measured dependence is referred to as the transfer characteristic, which shows that the current increases by about five orders of magnitude as compared to the structure of a one-layer polymer film (in this case, the currents are small and amount to less than $10 \mathrm{fA}$ ). The shape of the resultant curve indicates that it is electrons which are carriers responsible for charge transport. Indeed, an increase in current is observed for positive values of potential across the shutter.
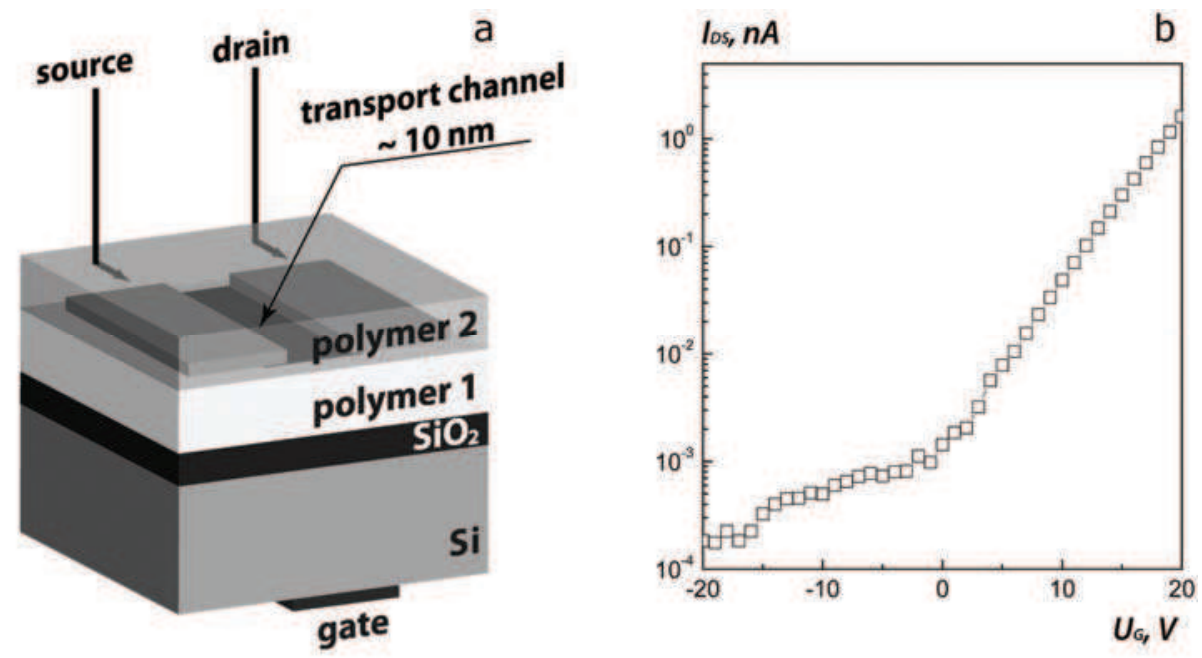

Fig. 5. Organic field-effect transistor based on two polymer films interface as a transport layer (a) and its transfer characteristic (b).

Furthermore, it was also found that it is possible to control the conductivity along the interface by the external electric field using the field-effect transistor circuit. The currentvoltage characteristics obtained in this experimental configuration allowed for additional 
estimates of the charge carrier mobility (Horowitz, 1998). The calculation was performed by the formula:

$$
I_{d}=\frac{Z}{L} \mu C_{i}\left(V_{g}-V_{t}\right) V_{d}
$$

where $I_{d}$ is the drain-source current; $Z$ and $L$ are the width and length of the conducting channel, respectively; $\mu$ is the mobility; $C_{i}$ is the dielectric layer capacitance; and $V_{g}, V_{d}$, and $V_{t}$ are the gate, source, and threshold voltages, respectively. The mobility calculated by formula (3) was $\mu=4 \cdot 10^{-2} \mathrm{~cm}^{2} \mathrm{~V}^{-1} \mathrm{~s}^{-1}$, and this agrees well with the earlier obtained value.

The temperature dependences of the conductivity measured for two different experimental configurations. Using the two-probe method we obtain the conductivity increases with the temperature. This behavior of the current-voltage characteristic can be attributed to the charge carrier injection from the metal electrodes into the polymer film limited by Schottky barrier. This conclusion agrees with the above analysis of the current-voltage characteristics (Salikhov et al., 2008). This means that the two-electrode method does not allow one to estimate the true electric conductivity along the interface between two polymer films. In view of this circumstance, the four-probe measurement of the conductivity was held. As the temperature increases, the electric conductivity decreases, possibly indicating its metal type. We note that a similar difference in the behavior of the conductivity temperature curves measured by the two- and four-probe methods was observed in the investigation of the properties of the quasi-two-dimensional electron gas formed along the $\mathrm{LaAlO}_{3}-\mathrm{SrTiO}_{3}$ and TTF-TCNQ interfaces (Kirtley \& Mannhart, 2008).

\section{Sensory properties}

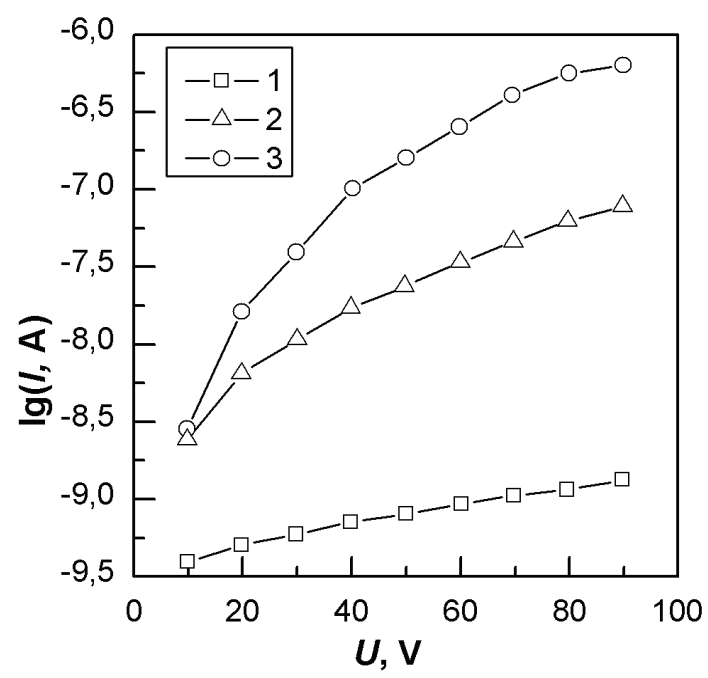

Fig. 6. Current-voltage characteristics of structures with aluminum electrodes, measured under different conditions: 1) with air relative humidity of $20 \%$; 2 ) in ethanol vapor; 3) with relative humidity of $80 \%$ 
The following are the sensory properties of samples with interface between two dielectric polymer films (Salikhov et al, 2009). In order to explain what part of a specimen is sensitive to a change in humidity (interface layer at the boundary of two polymer films or bulk material), the current-voltage $(I-V)$ characteristics were measured for specimens with a single film and lower arrangement of paired electrodes. In this case, humidity does not affect conductivity. As the results of measurements have shown, the greatest change in conductivity occurs with arrangement of electrodes between polymer layers.

$I-V$ characteristics are presented in Fig. 6 for a specimen with aluminum electrodes measured under different conditions. Curve 1 corresponds to measurement in an open atmosphere with $20 \%$ relative humidity. With an increase in relative humidity to $80 \%$ (curve 3), conductivity increases by three orders of magnitude. Curve 2 was obtained in ethanol $\mathrm{C}_{2} \mathrm{H}_{5} \mathrm{OH}$ vapor, whose molecule also contains a hydroxyl group. In this case, conductivity differs by two orders of magnitude from the original.

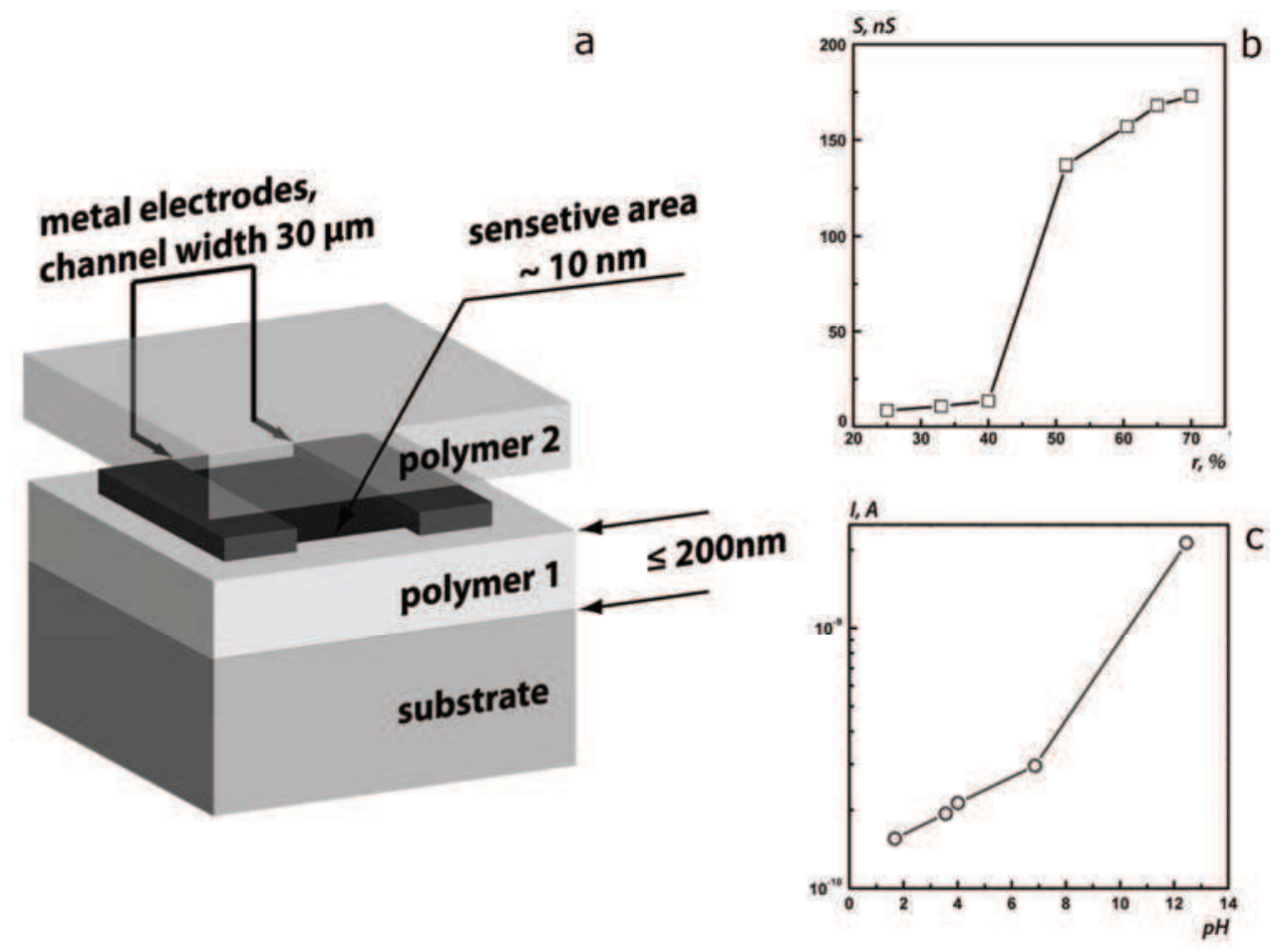

Fig. 7. Sensor on the base of two polymer films interface (a) and its characteristics:

dependence of conductivity on air relative humidity $r(b)$, dependence of current with a fixed voltage on $\mathrm{pH}$ index (c)

The next stage of the work (Salikhov et al., 2011) was creation of an acting model of a sensor for air relative humidity (see Fig. 7a). The current-voltage characteristics of the structures obtained were measured with different amounts of atmospheric humidity. A dependence of conductivity on relative humidity $r$ was constructed on the basis of measured curves, and it 
is presented in Fig. 7b. As follows from Fig. $7 b$, an increase in humidity affects the increase in specimen conductivity. It is possible to separate three sections on the curve in different ranges of humidity values. With relatively low humidity $(25-40 \%)$, there was little increase in conductivity. The next range (40-50\%) corresponds to a sharp increase in conductivity. With a further increase in humidity $(50-70 \%)$, there is an insignificant increase in conductivity with subsequent saturation. Rapid response sensors for relative humidity were studied. It follows from experiments that the response time is not more than $5 \mathrm{sec}$ (see Fig. 8). Most likely, this time corresponds to diffusion levelling with rapid opening of a cover beneath which there is a sensor with increased humidity of $80 \%$, with $20 \%$ humidity for the surrounding air. Use as dopants of substances whose molecules do not contain a hydroxyl group has demonstrated that in this case there is no change in conductivity. Thereby the selectivity has been demonstrated for the interface between polymer films to substances with hydroxyl groups. By using this unique feature of a transport layer, the experimental specimens obtained may be used as multiphase sensors for determining the hydrogen index (pH-meter), and this has been done by experiment. Compositions with a known $\mathrm{pH}$ value were added successively to a specimen. Then the current voltage characteristics were measured. The results obtained are shown in Fig. 7c in the form of a dependence of the current with the same fixed voltage on $\mathrm{pH}$ value. It follows from Fig. 3c that there is an increase in conductivity with a reduction in acidity. This confirms the assumption made about the elective sensitivity towards the hydroxyl group. The use of new polymer materials opens extensive possibilities for creating chemical nano-sized sensors for different substances. The selectivity is achieved by adjusting the upper polymer layer.

To explain the high conductivity over the interface between organic materials the various physical models were proposed. In (Nikitenko et al., 2010) the conductivity is due to the rather high surface density of geminate pairs formed at the interface. Conditions are established wherein the transitions of a significant portion of charge carriers between molecules do not require thermal activation or tunneling.

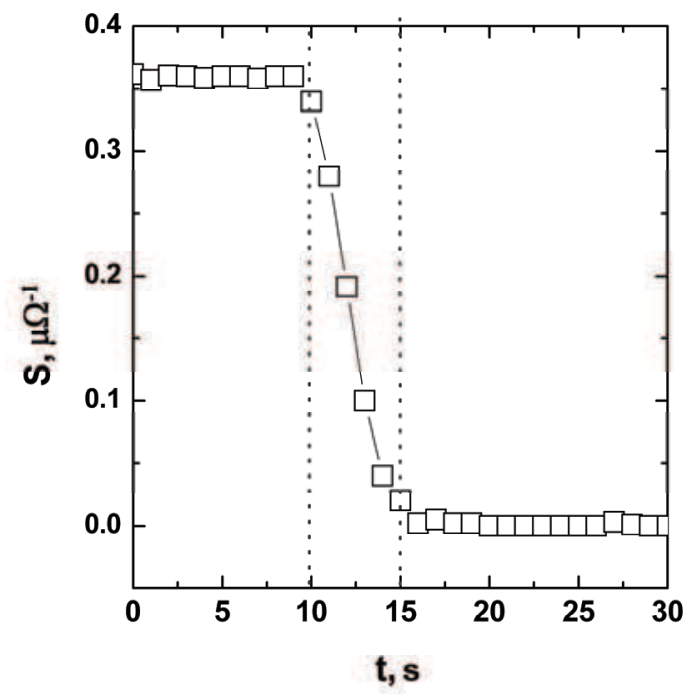

Fig. 8. Change in conductivity with time with a sharp reduction in humidity 
We can assume that the structure of the interface between two polymer layers differs from the equilibrium structure in the bulk of the sample. The experiments described in (Schefefold et al., 1996) show that polymers with side functional groups must orient themselves toward the interface between these groups. The latter may lead to selective ordering of these groups at the surface and to an increase in their local concentration due to close stacking. In the polymers studied here, the phthalide group that can be appropriately oriented relative to the film surface plays the role of the side group (see Fig. 9). In principle, this conclusion does not contradict the assumption of the possibility of formation of localized levels of the transient layer with the participation of side phthalide groups of polymer molecules, which are electron traps according to (Johansson et al., 1994)

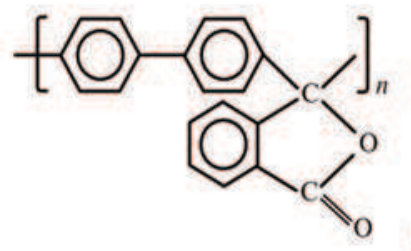

a

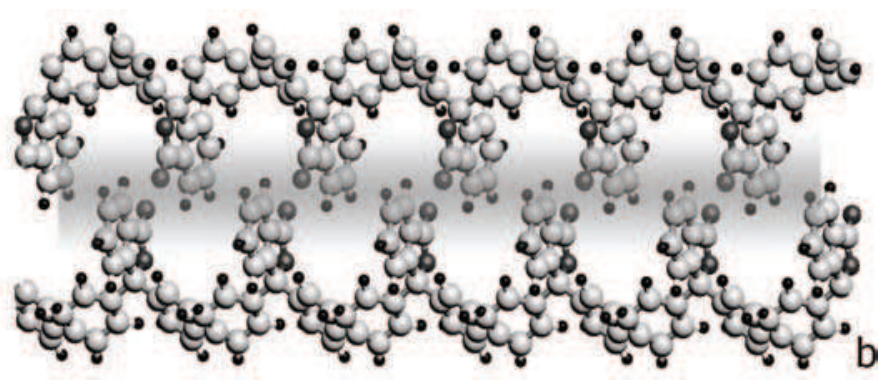

Fig. 9. Structural formula of poly(diphenylenephthalide)'s monomer (a) and threedimensional model image of two polymer films interface (b)

In the framework of one of the models previously proposed in (Nakagawa, et al., 2006; Pauli \& Willmott, 2008; Popović et al., 2008), it was assumed that to form the two- dimensional electron gas at the interface between two insulators, dipole groups should be present on at least one of the surfaces in contact. From this point of view, it seems possible to use materials having functional groups with a high dipole moment. Polymers can be taken as such materials. It is well known that the side functional groups of polymers are predominantly oriented along the normal to the polymer-air interface (Fischer et al., 1998). A similar orientation should occur when one polymer film is formed on the surface of the other. As a result, a nanolayer can appear in the near interface region, which is formed by the side groups of polymer molecules.

\section{Conclusion}

The sharp increase (by three to five orders of magnitude) in the conductivity of the samples after the deposition of the second polymer layer can be explained as follows. A thin transition layer with a structure differing from the bulk structure is apparently formed at the interface between the two polymers. We can assume that the interface between polymer films facilitates preferred antiparallel orientation of phthalide side fragments with an appreciable dipole moment (Lachinov et al., 2000). Such an orientation increases the local "2D" concentration of electron traps (phthalide side groups), which does not contradict the results obtained in (Schefefold et al., 1996). The trapping of the excess charge injected from the electrodes to phthalide groups of poly(diphenylenephthalide) leads to the emergence of deep localized states in the polymer band gap (Duke \& Fabish, 1976; Lachinov et al., 2000). These states ensure a higher conductivity level and modifies the shape of $I-V$ curves upon 
the passage of current. Thus, our studies lead to the conclusion on the possibility of formation of a transport layer at the interface of two polymer films, which possesses an elevated conductivity.

To summarize, we have shown that the interface between two dielectric polymer films has an anomalously high conductivity and charge carrier mobility. The investigation data such as the presence of a pronounced interface between the polymer films, the metal type of the conductivity, and the presence of the field effect suggest the presence of the quasi-twodimensional electron gas at the interface between the polymer insulators. However, this statement requires additional investigations, first of all, at low temperatures. It is interesting that the values of the charge carrier parameters at room temperature were relatively lower than the parameters of the interfaces prepared based on inorganic materials (Ohtomo \& Hwang, 2004; Reyren et al., 2007). Probably, this is attributed to the high defect content of the polymer-polymer interface due to the imperfect technology used in this interface preparation. Although the technology should be improved, the advantages of the polymer organic interfaces in the manufacture of the electronic materials with unique electronic properties are apparent. These materials are very promising for the production of new electronic devices, including chemical sensors for environmental monitoring, alarm systems and automation.

\section{Acknowledgment}

This work was supported by the Russian Foundation for Basic Research (projects no. 0903 00616a and 1102 01445a).

\section{References}

Alves H.; Molinari A.S.; Xie H. \& Morpurgo A.F. (2008). Metallic conduction at organic charge-transfer interfaces. Nature Mater. Vol. 7, pp. 574-580 (July 2008), ISSN 14761122

Angelopoulos, M. (2001). Conducting polymers in microelectronics. IBM J. Res.Dev., Vol. 45, No. 1, (January 2001), pp. 57-75, ISSN 0018-8646

Bailey, R. A. \& Persaud, K. C. (2000). Sensing volatile chemicals using conducting polymer arrays, In: Polymer Sensors and Actuators, Osada,Y. \& De Rossi, D. E., (Eds), pp. 149181, Springer, Berlin.

Bassler H. (1993). Charge Transport in Disordered Organic Photoconductors a Monte Carlo Simulation Study. Phys. Status Solidi B. Vol. 175, Is. 1, pp. 15-56 (January 1993), ISSN 1521-3951

Brédas J. L.; Chance R. R.; Silbey R.; Nicolas G. \& Durand Ph. (1981). A nonempirical effective Hamiltonian technique for polymers: Application to polyacetylene and polydiacetylene. J. Chem. Phys. Vol. 75, Is.1, p. 255 (13 pages) (July 1981), ISSN 00219606

Bunakov, A. A.; Lachinov, A.N. \& Salikhov, R. B. (2003). Current-voltage characteristics of poly(diphenylenephthalide) thin films. Techn. Phys. Vol. 48, No. 5, pp. 626-630 (May 2009), ISSN 1063-7842

Collins P. G.; Bradley K.; Ishigami M. \& Zettl A. (2000). Extreme Oxygen Sensitivity of Electronic Properties of Carbon Nanotubes. Science Vol. 287, No. 5459, pp. 18011804 (March 2000), ISSN 0036-8075 
Dewar M.J.S.; Zoebisch E.G.; Healy E.F. \& Stewart J.J.P. (1985). Development and use of quantum mechanical molecular models. 76. AM1: a new general purpose quantum mechanical molecular model. J. Am. Chem. Soc. Vol. 107, Is. 13, pp. $3902-3909$ (June 1985), ISSN 0002-7863

Dimitrakopoulos, C.D. \& Malenfant, P. R. L. (2002). Organic thin film transistors for large area electronics. Adv. Mater., Vol. 14, Is. 2, pp. 99-117 (January 2002), ISSN 09359648

Duke, C. B., \& Fabish, T. J. (1976). Charge-Induced Relaxation in Polymers. Phys. Rev. Lett. Vol. 37, Is. 16, pp. 1075-1078 (October 1976), ISSN 0031-9007

Fischer, D. A. ; Mitchell, G. E. ; Yeh, A. T. \& Gland J.L. (1998). Functional group orientation in surface and bulk polystyrene studied by ultra soft X-ray absorption spectroscopy. Appl. Surface Sci., Vol. 133, Is. 1-2, pp. 58-64 (May 1998), ISSN 01694332

Gadiev R.M.; Lachinov, A. N.; Kornilov V.M.; Salikhov, R. B.; Rakhmeev, R. G. \& Yusupov A.R.(2010). Anomalously high conductivity along the interface of two dielectric polymers. JETP Lett. Vol. 90, No. 11, pp. 726-730 (February 2010), ISSN 0021-3640

Gaponik, N. P.; Shchukin, D. G.; Kulak, A. I. \& Sviridov, D. V. (1997). A polyaniline based microelectrochemical transistor with an electrocatalytic gate. Mendeleev Commun. Vol. 7, Is. 2, pp. 70-71, ISSN 0959-9436

Gerard, M.; Chaubey, A. \& Malhotra, B. D. (2002). Application of conducting polymers to biosensors. Biosens. Bioelectron., Vol. 17, Is. 5, (May 2002), pp. 345-359, ISSN 09565663

Grate, J. W. (2000). Acoustic wave microsensor arrays for vapor sensing. Chem. Rev. Vol. 100, Is. 7, pp. 2627-2648 (July 2000), ISSN 0009-2665

Halik M.; Klauk H.; Zschieschang U.; Schmid G.; Dehm C.; Schütz M.; Maisch S.; Effenberger F.;, Brunnbauer M. \& Stellacci F. (2004). Low-voltage organic transistors with an amorphous molecular gate dielectric. Nature. Vol. 431, pp. $963-$ 966 (October 2004), ISSN 0028-0836

Hangarter, C.M.; Bangar, M.; Mulchandani, A. \& Myung N.V. (2010). Conducting polymer nanowires for chemiresistive and FET-based bio/chemical sensors. J. Mater. Chem., Vol. 20, Is. 16, pp. 3131-3140 (February 2010), ISSN 0959-9428

Horowitz G. (1998). Organic fielf-effect transistors. Adv. Mater. Vol. 10, Is. 5, pp. 365-377 (March 1998), ISSN 0935-9648

Johansson N.; Lachinov A.; Stafström S.; Kugler T. \& Salaneck W.R. (1994). A theoretical study of the chemical structure of the non-conjugated polymer poly(3,3'-phthalidylidene-4,4'-biphenylene). Synth. Met. Vol. 67, Is. 1-3, pp. 319-322 (November 1994), ISSN 0379-6779

Josowicz, M. \& Janata, J. (1986). Suspended gate field-effect transistor modified with polypyrrole as alcohol sensor. Anal. Chem. Vol. 58, Is. 3, (March 1986), pp. 514-517, ISSN 0003-2700

Kirtley, J. R. \& Mannhart, J. (2008). Organic electronics: When TTF met TCNQ. Nature Mater. Vol. 7, pp. 520-521 (July 2008), ISSN 1476-1122

Kornilov V. M. \& Lachinov A. N. (1997). Electrical conductivity in the metal-polymer-metal system: The role of boundary conditions. JETP Lett. Vol. 52, No. 4, pp. 833-841 (April 1997), ISSN 0021-3640 
Lachinov A. N.; Zherebov A. Yu. \& Kornilov V. M. (1990). Anomalous electron instability of polymers due to uniaxial pressure. JETP Lett. Vol. 52, No. 2, pp. 103-106 (July 1990), ISSN 0021-3640

Lachinov, A. N.; Zagurenko, T. G.; Kornilov, V. M.; Fokin A.I.; Aleksandrov I.V. \& Valiev R.Z. (2000). Charge transfer in a metal-polymer-nanocrystalline metal system. Phys. Solid State. Vol. 42, No.10, pp. 1935-1941 (October 2000), ISSN 1063-7834

Lachinov, A. N. ; Salikhov, R. B. ; Bunakov A.A. \& Tameev, A. R. (2004). Charge carriers generation in thin polymer films by weak external influences. Non-Linear Optics, Quantum Optics. Vol. 32, No. 1-3, pp. 13-20, ISSN 1543-0537

Lachinov A. N. ; Kornilov V. M.; Zagurenko T.G. \& Zherebov A. Yu. (2006). On the high conductivity of nonconjugated polymers. JETP. Vol. 102, No. 4, pp. 640-645 (April 2006), ISSN 1063-7761

Lachinov A. N.; Zhdanov E.R.; Rakhmeev R.G.; Salikhov R.B. \& Antipin V.A. (2010). Modulation of optical absorption in poly(diphenylenephthalide) films near the switching threshold. Phys. Solid State. Vol. 52, No. 1, pp. 195-200 (January 2010), ISSN 1063-7834

McQuade, D. T.; Pullen, A. E. \& Swager, T. M. (2000). Conjugated polymer-based chemical sensors. Chem. Rev., Vol. 100, No. 7, (July 2000), pp. 2537-2574, ISSN 0009-2665

Musa I. \& Eccleston W. (1999). Electrical properties of polymer/Si heterojunctions. Thin Solid Films. Vol. 343-344, pp. 469-475 (April 1999), ISSN 0040-6090

Nakagawa, N.; Hwang, H. Y. \& Muller, D. A. (2006). Why some interfaces cannot be sharp. Nature Mat., Vol. 5, No. 3, pp. 204-209 (March 2006) ISSN 1476-1122

Nikitenko, V. R.; Tameev, A. R. \& Vannikov, A. V. (2010). The mechanism of metal conductivity over the interface between organic insulators. Semiconductors. Vol. 44, No. 2, pp. 211-217 (February 2010), ISSN 1063-7826

Ohtomo, A., \& Hwang, H. Y. (2004). A high-mobility electron gas at the LaAlO3/SrTiO3 heterointerface. Nature. Vol. 427, pp. 423-426 (January 2004), ISSN 0028-0836

Pauli, S. A. \& Willmott, P. R. (2008). Conducting interfaces between polar and non-polar insulating perovskites. J. Phys.: Condens. Matter., Vol. 20, No. 26, 264012 (July 2008), ISSN 0953-8984

Polk, B. J.; Potje-Kamloth, K.; Josowicz, M. \& Janata, J. (2002). Role of protonic and charge transfer doping in solid-state polyaniline. J. Phys. Chem. Vol. 106 (44), pp. 1145711462 (November 2002), ISSN 1520-6106

Popović Z. S.; Satpathy S. \& Martin R. M. (2008). Origin of the two-dimensional electron gas carrier density at the LaAlO3 onSrTiO3 interface. Phys. Rev. Lett. Vol. 101, Is. 25, p. 256801 (4 pages) (December 2008), ISSN 0031-9007

Potje-Kamloth, K. (2001). Conducting polymer-based Schottky barrier and heterojunction diodes and their sensor application, In: Handbook of Surfaces and Interfaces of Materials, Nalwa, H. S., (Ed), Vol. 5, pp. 445-494, Academic, San Diego

Qi, P; Vermesh O.; Grecu M.; Javey A.; Wang Q.; Dai H.; Peng S. \& Cho K. J. (2003). Toward large arrays of multiplex functionalized carbon nanotube sensors for highly sensitive and selective molecular detection. Nano Lett. Vol.3, Is. 3, pp. 347-351 (March 2003), ISSN 1530-6984

Qiu L.; Lee W. H.; Wang X.; Kim J. S.; Lim J.A.; Kwak D.; Lee S. \& Cho K. (2009). Organic thin-film transistors based on polythiophene nanowires embedded in insulating polymer. Adv. Mater., Vol. 21, Is. 13, pp. 1349-1353 (April 2009), ISSN 0935-9648 
Reyren N.; Thiel S.; Caviglia A. D.; Kourkoutis L. F.; Hammerl G.; Richter C.; Schneider C. W.; Kopp T.; Rüetschi A.-S.; Jaccard D.; Gabay M.; Muller D. A.; Triscone J.-M. \& Mannhart J. (2007). Superconducting Interfaces Between Insulating Oxides. Science. Vol. 317, pp. 1196-1199 (August 2007), ISSN 0036-8075

Richert R.; Pautmeier L. \& Bassler H. (1989). Diffusion and drift of charge carriers in a random potential: Deviation from Einstein's law. Phys. Rev. Lett. Vol. 63, Is. 5, pp. 547-550 (July 1989), ISSN 0031-9007

Salazkin S. N.; Shaposhnikova V. V.; Machulenko L. N., Gileva N. G., Kraikin V. A. \& Lachinov A. N. (2008). Synthesis of polyarylenephthalides prospective as smart polymers. Polymer Sci. A. Vol. 50, No. 3, pp. 243-259 (March 2008), ISSN 0965-545X

Salleo A.; Chen T. W.; Völkel A. R.; Wu Y.; Liu P.; Ong B. S. \& Street R. A. (2004). Intrinsic hole mobility and trapping in a regioregular poly(thiophene). Phys. Rev. B. Vol. 70, p. 115311 (10 pages) (September 2004), ISSN 1098-0121

Salikhov R.B.; Lachinov A.N. \& Rakhmeyev R.G. (2007a). Electrical properties of heterostructure Si/poly(diphenylenephthalide)/Cu. J. Appl. Phys. Vol. 101, Is. 5, p. 053706 (5 pages) (March 2007), ISSN 0021-8979

Salikhov R.B.; Lachinov A.N. \& Rakhmeyev R.G. (2007b). Mechanisms of conductivity in metal-polymer-Si thin film structures. Mol. Cryst. Liq. Cryst. Vol. 467, pp. 85-92, ISSN 1542-1406

Salikhov R.B.; Lachinov A.N. \& Bunakov A.A. (2007c). Charge transfer in thin polymer films of polyarylenephthalides. Phys. Solid State. Vol. 49, No. 1, pp. 185-189 (January 2007), ISSN 1063-7834

Salikhov, R. B.; Lachinov, A. N. \& Rakhmeev, R. G. (2007d) Conduction mechanisms in silicon-polymer-metal heterostructures. Semiconductors. Vol. 41, No. 10, pp. 11651169 (October 2007), ISSN 1063-7826

Salikhov, R. B.; Lachinov, A. N. \& Rakhmeev, R. G. (2008). Transport layer at the boundary of two polymer films. Techn. Phys. Lett., Vol. 34, No. 6, pp. 495-497, ISSN 1063-7850

Salikhov, R. B.; Lachinov, A. N.; Kornilov V.M. \& Rakhmeev, R. G. (2009a). Properties of the transport layer formed at the interface between two polymer films. Techn. Phys. Vol. 54, No. 4, pp. 575-579 (April 2009), ISSN 1063-7842

Salikhov R.B.; Lachinov A.N.; Rakhmeyev R.G. ; Gadiev R.M.; Yusupov A.R. \& Salazkin S.N. (2009b). Chemical sensors based on nano-polymer films. Measurement Techniques. Vol. 52, No. 4, pp. 427-431 (April 2009), ISSN 0543-1972

Salikhov R.B.; Lachinov A.N.; Rakhmeyev R.G. \& Gadiev R.M. (2011). High Conductivity of the Interface Between Two Dielectric Polymer Films. Mol. Cryst. Liq. Cryst. Vol. 535, Is. 1, pp. 74-81, ISSN 1542-1406

Schefefold, F.; Budkowski, A.; Steiner, U.; Eiser E.; Klein J. \& Fetters L.J. (1996). Surface phase behavior in binary polymer mixtures. II. Surface enrichment from polyolefin blends. J. Chem. Phys., Vol. 104, Is. 21, p. 8795 (12 pages) (June 1996), ISSN 00219606

Sundgren, H.; Lundstroem, I.\& Vollmer, H. (1992). Chemical sensor arrays and abductive networks. Sens.Actuat. B Vol. 9, Is. 2, pp. 127-131 (August 1992), ISSN 0925-4005

Zykov B.G.; Baydin V.N.; Bayburina Z.Sh.; Timoshenko M.M.; Lachinov A.N. \& Zolotuchin M.G. (1992). Valence electronic structure of phthalide-based polymers. J. Electron. Spectrosc. Rel. Phenom. Vol. 61, Is. 1, pp. 123-129 (December 1992), ISSN 0368-2048 


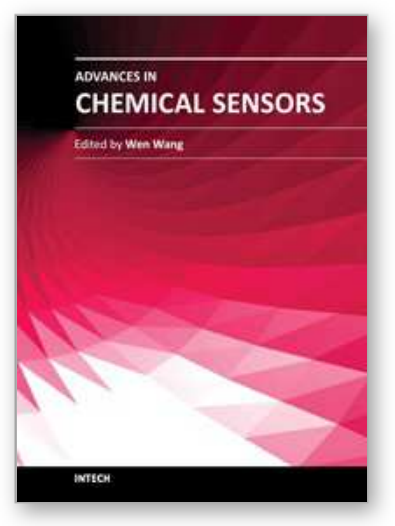

\author{
Advances in Chemical Sensors \\ Edited by Prof. Wen Wang
}

ISBN 978-953-307-792-5

Hard cover, 358 pages

Publisher InTech

Published online 20, January, 2012

Published in print edition January, 2012

The chemical sensor plays an essential role in the fields of environmental conservation and monitoring, disaster and disease prevention, and industrial analysis. A typical chemical sensor is a device that transforms chemical information in a selective and reversible way, ranging from the concentration of a specific sample component to total composition analysis, into an analytically useful signal. Much research work has been performed to achieve a chemical sensor with such excellent qualities as quick response, low cost, small size, superior sensitivity, good reversibility and selectivity, and excellent detection limit. This book introduces the latest advances on chemical sensors. It consists of 15 chapters composed by the researchers active in the field of chemical sensors, and is divided into 5 sections according to the classification following the principles of signal transducer. This collection of up-to-date information and the latest research progress on chemical sensor will provide valuable references and learning materials for all those working in the field of chemical sensors.

\title{
How to reference
}

In order to correctly reference this scholarly work, feel free to copy and paste the following:

Renat Salikhov and Aleksey Lachinov (2012). Polymer Thin Film Chemical Sensors, Advances in Chemical Sensors, Prof. Wen Wang (Ed.), ISBN: 978-953-307-792-5, InTech, Available from:

http://www.intechopen.com/books/advances-in-chemical-sensors/polymer-thin-film-chemical-sensors

\section{INTECH}

open science | open minds

\section{InTech Europe}

University Campus STeP Ri

Slavka Krautzeka 83/A

51000 Rijeka, Croatia

Phone: +385 (51) 770447

Fax: +385 (51) 686166

www.intechopen.com

\section{InTech China}

Unit 405, Office Block, Hotel Equatorial Shanghai

No.65, Yan An Road (West), Shanghai, 200040, China

中国上海市延安西路65号上海国际贵都大饭店办公楼 405 单元

Phone: +86-21-62489820

Fax: $+86-21-62489821$ 
(C) 2012 The Author(s). Licensee IntechOpen. This is an open access article distributed under the terms of the Creative Commons Attribution 3.0 License, which permits unrestricted use, distribution, and reproduction in any medium, provided the original work is properly cited. 